\title{
Microscopic Richtmyer-Meshkov instability under strong shock
}

\author{
Cite as: Phys. Fluids 32, 024109 (2020); doi: 10.1063/1.5143327 \\ Submitted: 22 December 2019 - Accepted: 5 February 2020 • \\ Published Online: 24 February 2020
}

\begin{abstract}
Pengyue Sun, ' Juchun Ding,, ${ }^{1, a)}$ (D) Shenghong Huang, ${ }^{1}$ (D) Xisheng Luo,' (D) and Wan Cheng ${ }^{2}$
AFFILIATIONS

${ }^{1}$ Department of Modern Mechanics, University of Science and Technology of China, Hefei 230026, China

${ }^{2}$ Mechanical Engineering, Physical Science and Engineering Division, King Abdullah University of Science and Technology, Thuwal 23955-6900, Saudi Arabia
\end{abstract}

a) Author to whom correspondence should be addressed: djc@mail.ustc.edu.cn

\begin{abstract}
The microscopic-scale Richtmyer-Meshkov instability (RMI) of a single-mode dense-gas interface is studied by the molecular dynamics approach. Physically realistic evolution processes involving the non-equilibrium effects such as diffusion, dissipation, and thermal conduction are examined for different shock strengths. Different dependence of the perturbation growth on the shock strength is found for the first time. Specifically, the amplitude growths for cases with relatively lower shock Mach numbers $(\mathrm{Ma}=1.9,2.4,2.9)$ exhibit an evident discrepancy from a very early stage, whereas for cases with higher Mach numbers $(\mathrm{Ma}=4.9,9.0,16.0)$, their amplitude variations with time match quite well during the whole simulation time. Such different behaviors are ascribed to the viscosity effect that plays a crucial role in the microscale RMI. The compressible linear theory of Yang et al. ["Small amplitude theory of Richtmyer-Meshkov instability," Phys. Fluids 6(5), 1856-1873 (1994)] accounting for the viscosity dissipation provides a reasonable prediction of the simulated linear growth rate. Furthermore, a modified compressible nonlinear model [Q. Zhang et al., "Quantitative theory for the growth rate and amplitude of the compressible RichtmyerMeshkov instability at all density ratios," Phys. Rev. Lett. 121, 174502 (2018)] considering both the viscosity effect and the corrected linear growth rate is proposed, which gives an excellent forecast of the linear and nonlinear growths of the present microscale RMI.
\end{abstract}

Published under license by AIP Publishing. https://doi.org/10.1063/1.5143327

\section{INTRODUCTION}

The Richtmyer-Meshkov instability (RMI) ${ }^{1,2}$ arises when a corrugated interface separating two fluids of different properties is impulsively accelerated by a shock wave. After the shock impact, initial corrugations on the interface are strongly stretched and, as a consequence, the perturbation amplitude grows continuously with time and finally triggers the flow transition to turbulent mixing. In the past few decades, the RMI has been a subject of intensive research due to its crucial role in applications of a broad range of scales ranging from millimeters [e.g., inertial confinement fusion (ICF) $]^{3}$ to light years (e.g., supernova explosion). ${ }^{4}$ Previous studies on RMI mainly focused on the instability development at a laboratory length scale, and numerous theoretical models were proposed to estimate the perturbation growth from linear ${ }^{1,5,6}$ to nonlinear stages. ${ }^{7-10}$ Recently, the validity of the existing models has been thoroughly examined by a set of high-fidelity experimental results.
The RMI at microscales under a strong shock is a more realistic approximation of the hydrodynamic instability in ICF, and thus the related research is very desirable and attractive. However, the microscopic scale and the strong shock condition bring great challenges to the experiments, simulations, and theoretical analyses on this subject. Experimentally, it is very difficult to capture the instability evolution process at small scales. ${ }^{12,13}$ Numerically, traditional hydrodynamic methods, which are widely applied in the study of RMI at a laboratory length scale, are not applicable to the simulation of microscopic RMI for requiring known equations of state and appropriate settings on a numerical scheme and a grid structure. Moreover, the interface evolution at microscales involves strong non-equilibrium effects including molecular diffusion, viscosity, and heat conduction, for which the traditional hydrodynamic methods cannot provide satisfactory results.

The molecular dynamics (MD) approach, which is based on the track of atom movements, has been demonstrated to provide an unmatched capacity in reproducing microscopic flows with strong 
non-equilibrium effects. In recent years, the MD approach has been successfully applied to the RMI research. A straightforward application of the MD method to simulate a heavy/light liquid interface subjected to a shock wave in cylindrical and planar geometries was reported by Zhakhovskii et al., ${ }^{15}$ and detailed flow structures with atomic resolution were captured. The MD simulation of a solid interface impacted by a strong shock was performed by Zybin et al., ${ }^{16}$ and the dependence of the instability evolution on the shock strength and orientation was discussed. Large-scale nonequilibrium $\mathrm{MD}$ simulations of a roughened $\mathrm{Cu} /$ vacuum $(\mathrm{Cu} / \mathrm{Ne})$ interface impacted by a planar shock were conducted by Germann et al., ${ }^{17}$ and rich phenomena were observed. In addition, the propagating behavior and the wave structure for a strong cylindrical or spherical shock imploding in a convergent geometry have been carefully investigated via the MD method by Liu et al. ${ }^{18}$ Recently, the RMI of a single-mode lithium/hydrogen interface under extreme shock conditions is numerically studied by the MD simulation incorporated with the electron force field model, and the ionization effect was found to play a significant role in the instability growth. ${ }^{19}$ In addition, several published works using the MD method studied the role of ejecta production and mostly focused on the velocity and size distribution of the ejected material. ${ }^{20,21}$ An alternative atomistic method, Direct Simulation Monte Carlo (DSMC) simulation, was also demonstrated to be able to investigate the hydrodynamic instabilities. $^{22,23}$

The former studies mainly considered the RMI of the multiphase material interface, and the analyses were restricted to qualitative descriptions of the interface evolution. So far, the microscopic RMI of a dense-gas interface under a strong shock, which is a realistic approximation of the hydrodynamic instability in ICF, has never been studied, and the flow regimes (e.g., the shock intensity influence and the spatial scale effect on the instability growth) are not yet understood. This motivates the present study. In this work, the RMI of a single-mode gaseous interface between dense helium $(\mathrm{He})$ and argon (Ar) accelerated by a shock wave is simulated by the classical MD method. Various incident shock (IS) strengths are considered to examine the influence of the shock intensity on the instability growth. The validity of the existing models in predicting microscopic RMI will be assessed. In addition to the well-known flow mechanisms such as baroclinic vorticity and pressure perturbation, ${ }^{24-26}$ new physical regimes for the microscale RMI will be discussed and modeled.

\section{NUMERICAL METHODS}

The microscopic RMI of a single-mode helium/argon gaseous interface is simulated by the Large-scale Atomic/Molecular Massively Parallel Simulator (LAMMPS) code, which was originally distributed by the Sandia National Laboratory. ${ }^{27}$ The LAMMPS solver has been widely used and also shown a good performance in studies of shock propagation and shock-induced interfacial instability. ${ }^{19,28,29}$ For the classical MD method, the atom is treated as a whole and its movement follows Newton's second law,

$$
\boldsymbol{F}_{i}=m_{i} \nabla U
$$

Here, $m_{i}$ denotes the mass of molecule $i$ and $\boldsymbol{F}_{i}$ is the sum of forces acting on molecule $i$, which can be obtained by the gradient of potential function $U$. Once the $\boldsymbol{F}_{i}$ is obtained, the molecule acceleration $\boldsymbol{a}_{i}$, velocity $\boldsymbol{v}_{i}$, and position $\boldsymbol{r}_{i}$ are available by the mechanical laws,

$$
\boldsymbol{F}_{i}=m_{i} \boldsymbol{a}_{i}, \quad \boldsymbol{v}_{i}=\boldsymbol{v}_{i}^{0}+\boldsymbol{a}_{i} t, \quad \boldsymbol{r}_{i}=\boldsymbol{r}_{i}^{0}+\boldsymbol{v}_{i}^{0} t+\frac{1}{2} \boldsymbol{a}_{i} t^{2} .
$$

Then, macroscopic physical quantities such as temperature, pressure, and density can be readily obtained via statistical methods.

The pair interactions for $\mathrm{He}-\mathrm{He}, \mathrm{He}-\mathrm{Ar}$, and $\mathrm{Ar}-\mathrm{Ar}$ are described by the Lennard-Jones (LJ) potential, ${ }^{30}$ which is formulated as

$$
U_{i j}=4 \varepsilon\left[\left(\frac{\sigma}{r_{i j}}\right)^{12}-\left(\frac{\sigma}{r_{i j}}\right)^{6}\right], \quad r_{i j}<r_{c},
$$

where the LJ cutoff radius $r_{c}$ is set to be $10 \AA . \sigma_{A r}=3.405 \AA$ and $\varepsilon_{A r}=0.01032 \mathrm{eV}$ are adopted for the $\mathrm{Ar}-\mathrm{Ar}$ interaction and $\sigma_{H e}$ $=2.28 \AA$ and $\varepsilon_{\mathrm{He}}=0.000876 \mathrm{eV}$ for He-He. ${ }^{30}$ The parameters for the interaction between $\mathrm{He}$ and $\mathrm{Ar}$ are calculated by the customary Lorentz-Berthelot mixing rules,

$$
\sigma_{H e-A r}=\frac{\sigma_{H e}+\sigma_{A r}}{2}, \quad \varepsilon_{H e-A r}=\sqrt{\varepsilon_{H e} \cdot \varepsilon_{A r}} .
$$

The computational setting for a planar shock colliding with a sinusoidally perturbed helium/argon interface is shown in Fig. 1. The simulation box has a volume of $9500 \times 800 \times 20 \AA^{3}(x \times y \times z)$ and is filled with $4.8 \times 10^{6}$ atoms initially. An incident planar shock is produced by a solid piston moving from left to right at a constant velocity. The regions on the left and right sides of the interface are occupied by helium and argon atoms, respectively. In the Cartesian coordinate system, the single-mode interface is described as $x=x_{0}$ $+a_{0} \cos (k y)$, with $k$ standing for the wavenumber, $a_{0}$ for the initial perturbation amplitude, and $x_{0}$ for the mean position of the initial interface. Periodic boundary conditions are adopted in the $y$ and $z$ directions. The time step is set to be 0.0001 ps throughout the computation. In order to save the computational time and memory, the present domain is automatically enlarged along the $x$ direction as the shock wave reaches the box boundary and, accordingly, the atom number is increased gradually from 4.8 to $11 \times 10^{6}$. Note that in the MD simulation, the computational domain is just filled with atoms without a spatial discretization. Hence, there is no grid convergence problem here.

The simulation system is first relaxed for a short period of 1 ps with the isothermal-isobaric ensemble (NPT), for which the absolute temperature and the pressure are fixed at $300 \mathrm{~K}$ and 2500 bars, respectively. Then, a thermodynamically equilibrium state is obtained with the densities of helium and argon being $0.24 \mathrm{~g} / \mathrm{cm}^{3}$

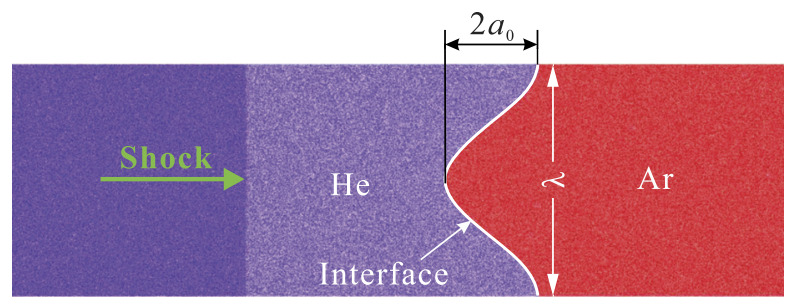

FIG. 1. Schematic of the computational setting for a planar shock wave interacting with a single-mode helium/argon interface. The $a_{0}$ refers to the initial amplitude of the perturbation and $\lambda$ to the wavelength. 
and $1.26 \mathrm{~g} / \mathrm{cm}^{3}$, respectively. It should be pointed out that the present gaseous helium and argon are in moderate-energy-density state for which any ideal equation of state loses its validity, and the density of each gas is calculated by dividing the mass by volume. Later, the solid piston starts moving from left to right at a constant velocity $\left(v_{p}\right)$ under the microcanonical ensemble framework (NVE) where the particle number, volume, and energy of the system are fixed. The moving piston compresses continuously the helium and eventually produces a planar shock wave with a certain strength. In order to prevent the gas diffusion across the interface before the shock-interface collision, a spurious solid wall is set along the interface and then removed just before the shock arrival. Note that in our previous work, ${ }^{19}$ the electronic effects on the instability growth have been specially discussed. Nevertheless, in that work, the effects introduced by the microscale were not clarified since the coexistence of electronic effects and microscale effects complicates the flow analysis. In the present MD simulations, we close the ionization switch to eliminate electronic effects such that the microscale effects can be analyzed in detail.

\section{RESULTS AND DISCUSSION}

The sinusoidal helium/argon interface with an amplitude-towavelength ratio of $a_{0} / \lambda=0.05\left(a_{0}=40 \AA\right.$ and $\left.\lambda=2 \pi / k=800 \AA\right)$ subjected to a planar shock of different strengths (corresponding to different piston velocities) is considered. Detailed parameters corresponding to the initial conditions for each case are listed in Table I, where the post-shock quantities are also given. The postshock temperature is calculated by statistical mechanics $\Sigma \frac{1}{2} m v^{2}$ $=\frac{3}{2} N k_{b} T$, where the translational kinetic energy of the post-shock flow should be ignored. The sound speed of the pre-shock helium is evaluated to be $1442 \mathrm{~m} / \mathrm{s}(14.4 \AA / \mathrm{ps})$ by computing the dynamic structure factor, ${ }^{31}$ and hence the incident shock Mach number can be readily obtained. Special attention is paid to the influence of the incident shock strength on the instability development. Even though the spatial scale of the instability considered here is much smaller than that of the ICF target due to the limited computational resources, the results obtained can clearly reveal the scale effect on the hydrodynamic instability.

\section{A. Flow features and interface evolution}

Representative atom distribution images illustrating the singlemode interface development under three shock strengths (cases 2,
4, and 6) are displayed in Fig. 2. The instability developments for the three cases are similar, and we shall take case 2 as an example to detail the evolution process. Temporal origin in this work is defined as the moment when the incident shock just traverses the sinusoidal interface. The time is normalized as $\tau=k v_{0} t$, with $v_{0}$ being the linear growth rate. Generally, the whole evolution processes of the waves and the interface are analogous to those of macroscopic-scale shocktube experiments. ${ }^{11,32,33}$ At the beginning $(\tau=-0.12)$, a planar incident shock (IS) is well produced in He due to the continuous compression by a moving piston and the initial interface (II) presents a distinct single-mode morphology with no diffusion before the shock impact. The perfect initial conditions produced demonstrate the good feasibility and reliability of the numerical techniques for the shock and interface formations. As the incident shock passes across the initial interface, it bifurcates into a downstream-moving transmitted shock (TS) and an upstream-moving reflected shock (RS). The transmitted and reflected shocks present a visible sine-like perturbation at the early stage and later recover gradually to be uniform. As time proceeds, the shocked interface (SI) moves forward with its perturbation amplitude growing persistently. Although gas diffusion across the interface is allowed after the shock-interface collision (i.e., the artificially embedded solid wall is removed from the interface), the evolving interface still presents distinct morphologies during the time of interest, which greatly facilitates the measurement of perturbation amplitude and the extraction of interfacial morphology. Since the MD approach is based on the track of atom motions, the present instability evolution naturally involves all non-equilibrium physical processes such as diffusion, dissipation, and thermal conduction, which cannot be accurately modeled by the traditional hydrodynamics methods. Hence, we claim that the present results are more physically accurate than the traditional hydrodynamics ones. Induced by the baroclinic vorticity distributed along the interface, the shocked interface deforms persistently, causing a continuous increment in perturbation amplitude. At the early stage, the distorting interface is symmetric and presents a perfect sinusoidal shape, which indicates a linear growth of the instability. As time proceeds, nonlinearity becomes pronounced and higher harmonics are generated gradually, producing asymmetric spike and bubble structures $(\tau=0.71)$. At late stages, the spikes start to roll up. It should be pointed out that the roll-up structure here is much less distinct than that of macroscopic $\mathrm{RMI}^{34,35}$ which is mainly ascribed to the intense molecular motion. For case 6 with a much stronger incident shock, the interface suffers a greater compression by the shock and subsequently presents a

TABLE I. Parameters corresponding to the initial conditions for each case. $v_{p}, v_{s}, v_{i}$, and $v_{t}$ are speeds ( $\left.\AA / p s\right)$ of the piston, the incident shock, the shocked interface, and the transmitted shock, respectively. $a_{0}$ and $\lambda$ are the amplitude and the wavelength of the initial perturbation, respectively. The superscript + denotes the post-shock state. $T_{1}\left(T_{2}\right)$ denotes the gas temperature on the left (right) side of the shocked interface.

\begin{tabular}{lrrrrrrrrrr}
\hline \hline Case & $v_{p}$ & \multicolumn{1}{c}{$v_{s}$} & $\mathrm{Ma}$ & $a_{0} / \lambda$ & $a_{0}^{+} / \lambda$ & $A^{+}$ & $T_{1}(\mathrm{~K})$ & $v_{i}$ & $T_{2}(\mathrm{~K})$ & $v_{t}$ \\
\hline 1 & 10 & 27.6 & 1.9 & 0.05 & 0.041 & 0.59 & 651.5 & 5.0 & 654.3 & 19.7 \\
2 & 15 & 34.1 & 2.4 & 0.05 & 0.039 & 0.56 & 917.6 & 7.8 & 995.5 & 24.9 \\
3 & 20 & 41.2 & 2.9 & 0.05 & 0.037 & 0.55 & 1255.8 & 10.7 & 1496.8 & 30.1 \\
4 & 40 & 70.4 & 4.9 & 0.05 & 0.035 & 0.52 & 3524.5 & 22.4 & 5325.0 & 50.9 \\
5 & 80 & 129.1 & 9.0 & 0.05 & 0.033 & 0.51 & 12856.4 & 46.4 & 23899.2 & 91.6 \\
6 & 150 & 230.2 & 16.0 & 0.05 & 0.031 & 0.51 & 44808.5 & 88.9 & 95215.6 & 159.9 \\
\hline \hline
\end{tabular}


Case 2
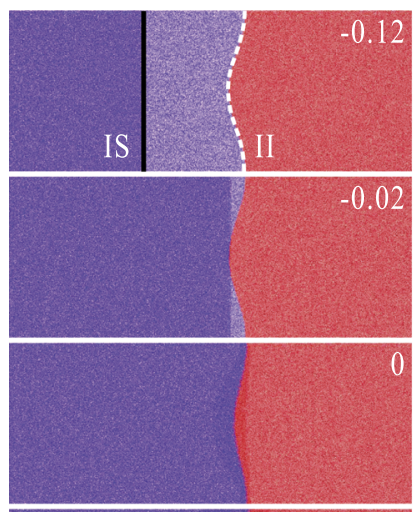

0.04
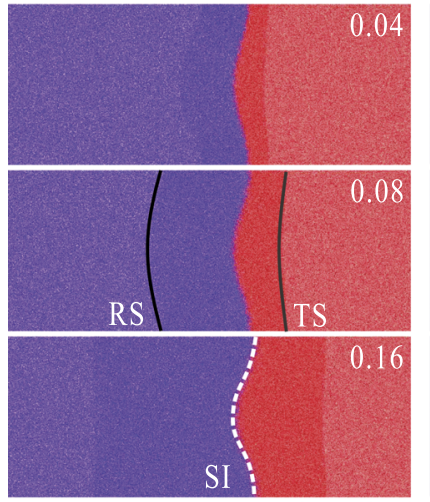

0.4

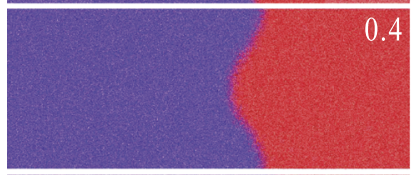

0.71

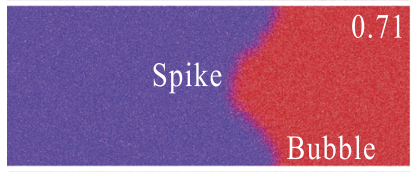

1.03
Case 4
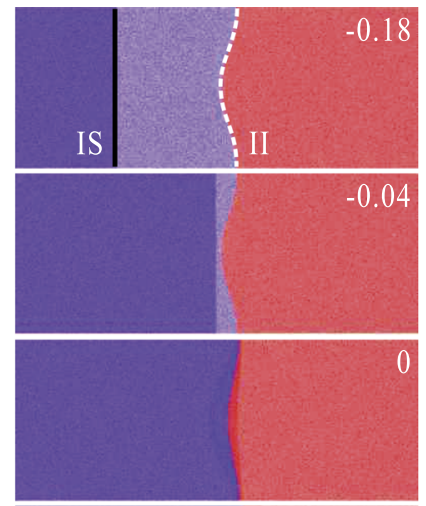

0.04
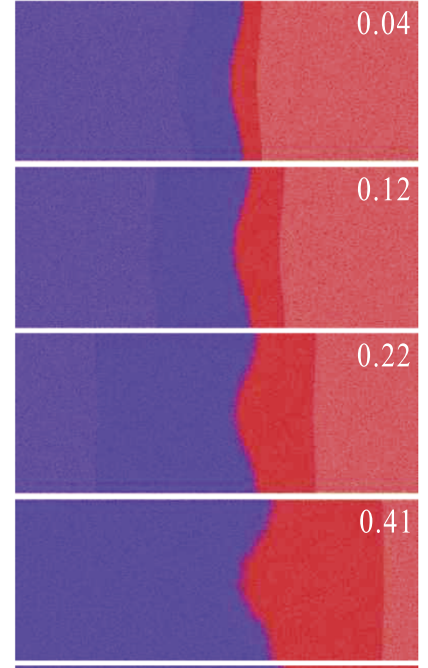

0.71

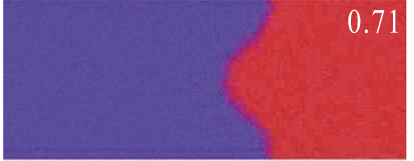

1.01
Case 6
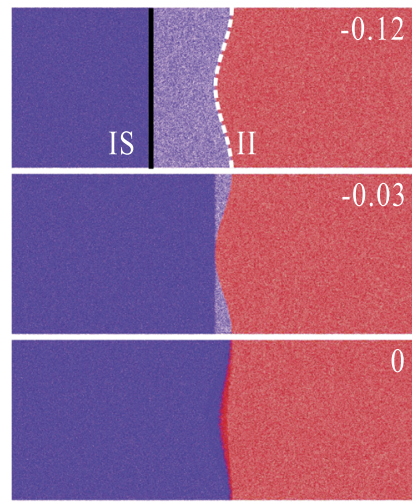

0.05

0.09

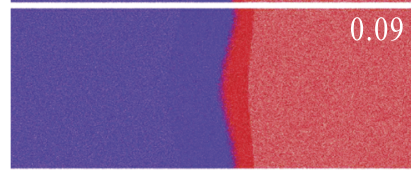

0.16
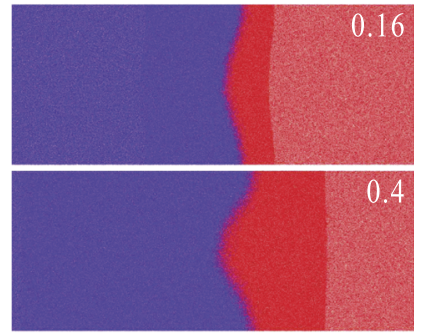

0.71

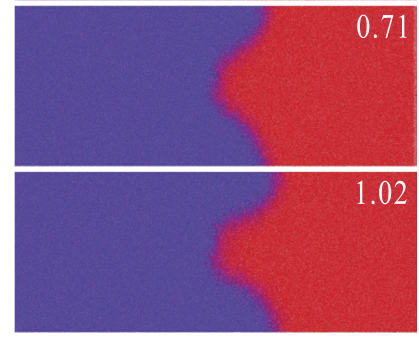

FIG. 2. Representative atom distribution snapshots showing the evolution of a single-mode helium/argon interface with a fixed amplitude-to-wavelength ratio $\left(a_{0} / \lambda=0.05\right)$ subjected to a planar shock of $\mathrm{Ma}=2.4$ (case 2), $\mathrm{Ma}=4.9$ (case 4), and $\mathrm{Ma}=16.0$ (case 6). IS, incident shock; II, initial interface; TS, transmitted shock; RS, reflected shock; and $\mathrm{SI}$, shocked interface. Numbers in the images denote the dimensionless time, which is normalized as $\tau=k v_{0} t$, with $v_{0}$ being the initial growth rate. quicker instability development. In addition, the transmitted shock propagates more slowly relative to the shocked interface, and thus the interface evolution suffers a longer-term influence by the pressure waves emitted from the disturbed transmitted shock. As a consequence, a considerably flat bubble structure is observed in case 6 $(\tau=1.02)$, which is similar to the result of Sadot $e t a l .{ }^{36}$ It should be stressed that the present study mainly concerns the microscale effect on the instability growth, and the ionization process is not considered although the post-shock temperatures for cases 5 and 6 actually exceed the critical temperature for ionization. ${ }^{3}$

Dimensionless variations of the perturbation amplitude vs time for all cases are plotted and compared with the theoretical predictions in Fig. 3(a). Note in this work, the interface boundary is determined by the middle position (i.e., $50 \%$ mass fraction) of the material layer and the perturbation amplitude is defined as a half of the distance between the tips of the spike and bubble (see Fig. 1). Here, the time-varying amplitude is scaled as $\alpha=k\left(a-a_{0}^{*}\right)$, with $a_{0}^{*}$ being the perturbation amplitude at $\tau=0$. Although each theoretical model in Fig. 3 gives different predictions for cases with different shock Mach numbers, the normalized results for these cases match quite well and show a very small discrepancy. Here, for a clear presentation, only the prediction of each model for case 1 is given. It is seen that the amplitude growths for cases 1-3 with relatively weaker incident shocks are almost identical at the very early stage $(\tau<0.4)$ but later exhibit an increasing discrepancy. In addition, the weaker the incident shock, the earlier the linear growth saturation. 

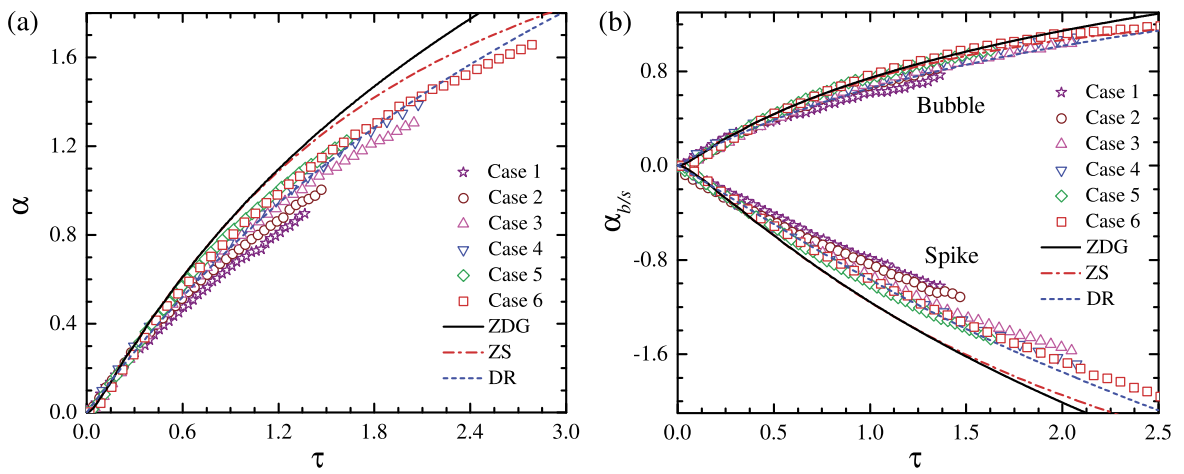

FIG. 3. Comparisons between the simulations and the model predictions for (a) the whole perturbation amplitude $(\alpha)$ and (b) the amplitudes of bubbles $\left(\alpha_{b}\right)$ and spikes $\left(\alpha_{s}\right)$. Note $\alpha=\left(\alpha_{b}+\alpha_{s}\right) / 2$. Symbols stand for the MD results and lines for the model predictions. Nonlinear models are $\mathrm{DR},{ }^{9} \mathrm{ZS},{ }^{7}$ and $\mathrm{ZDG}$.

Nevertheless, for cases 4-6 with stronger incident shocks, the amplitude variations with time for these cases match quite well during the whole simulation time. To the best of the authors' knowledge, such different responses of the instability growth to the shock strength increment are first found for the microscopic RMI. Although the influence of shock strength on the growth of RMI has been studied before, ${ }^{38-40}$ those works mainly considered the RMI at macroscopic scales through hydrodynamic simulations or experiments. For microscopic RMI, new flow regimes caused by non-quilibrium physical processes (well captured by the MD approach) play important roles and thus the influence of shock strength on the perturbation growth would be different from the macroscopic counterpart. The reliable data obtained provide a rare opportunity to examine the applicability of the existing theoretical models in predicting the microscopic RMI, for which there is no theory.

Recently, an accurate and robust compressible nonlinear model $^{10}$ (called the ZDG model) applicable to systems with arbitrary density ratios and strong incident shocks has been established based on the methods of two-point Padé approximation and asymptotic matching, which is written as

$$
v_{n l}^{c}\left(t, A, v_{\infty}^{c}\right)=v_{\text {lin }}^{c}(t, A) P_{3}^{2}\left(t, A, v_{\infty}^{c}\right) .
$$

Here, $v_{n l}^{c}$ refers to the compressible nonlinear growth rate, $v_{\text {lin }}^{c}$ to the compressible linear growth rate, ${ }^{41}$ and $v_{\infty}^{c}$ to the asymptotic growth rate of compressible linear theory. Although this compressible model has shown a good performance in predicting the linear and nonlinear growths of RMI under a wide range of shock Mach numbers, it overestimates the present MD result from a very early time. This is ascribed to the absence of viscosity effect in the theory of Zhang et al. ${ }^{10}$ because viscosity may play a crucial role in the microscopic RMI that usually presents a very low Reynolds number. ${ }^{42,43}$ For the RMI flow, a thin vorticity layer along the interface is produced by a baroclinic mechanism during the shock-interface interaction. ${ }^{44,45}$ This means that there is a sudden jump in the tangential velocity of the flow across the interface. For such a strong shear flow, viscosity would play a significant role in the vorticity transport and further influences the interface evolution. In the following analysis, we will verify this assumption.

The normalized variations of individual amplitudes of the bubble and spike are also shown in Fig. 3(b). The amplitude of the bubble (spike) is measured by taking the trajectory of the corresponding unperturbed interface as a moving reference. It is seen that the bubble growth remains almost identical for all cases (i.e., very weak dependence on the shock strength) and can also be reasonably predicted by all the existing models. This suggests a straightforward application of the previous theories to predict the bubble growth of microscopic RMI. Differently, the growth of the spike depends heavily on the incident shock strength for cases 1-3 but exhibits a very small discrepancy among cases $4-6$. The overestimation of the spike growth for cases $1-3$ is a major reason for the large deviation in the overall perturbation amplitude between the MD result and the model prediction. The present result can be explained by that viscous dissipation can evidently damp secondary instabilities such as Kelvin-Helmholtz (KH) instability at the roll-up of the spike ${ }^{46}$ and hence produces a more pronounced influence on the growth of the spike than the bubble.

\section{B. Evaluation of viscosity effect on microscale RMI}

In previous studies, ${ }^{42,43}$ to quantify the viscosity effect, dimensional quantities such as viscosity coefficient are introduced to the theoretical models. The authors believe that a nondimensionalized Reynolds number ( $\mathrm{Re}$ ) can better reveal the viscous effect and should be adopted in the viscous models. For this purpose, a Re defined by characteristic quantities of the RMI flow is proposed,

$$
\operatorname{Re}=v_{0} \lambda \frac{\left(\rho_{1}+\rho_{2}\right)^{2}\left(\sqrt{\rho_{1} \mu_{1}}+\sqrt{\rho_{2} \mu_{2}}\right)^{2}}{\rho_{1} \mu_{1} \rho_{2} \mu_{2}},
$$

where $\mu_{1}\left(\mu_{2}\right)$ and $\rho_{1}\left(\rho_{2}\right)$ are the dynamic viscosity and the density for fluid 1 (fluid 2), respectively, and $v_{0}$ is the growth rate at the linear stage. The dynamic viscosities and the densities of the post-shock fluids on both sides of the interface can be calculated by the statistical mechanics and are listed in Table II. It should be pointed out that the definition of Re, here, aims to facilitate the formulation of Eqs. (7) and (10), i.e., the dimensional quantities such as dynamics viscosity in these equations can be replaced by Re, which better characterizes the viscosity effect.

Figure 4 plots the variations of Re vs the piston velocity for all cases. Note that to enable a smooth fitting, the data from additional four cases $\left(v_{p}=30 \AA / \mathrm{ps}, 60 \AA / \mathrm{ps}, 100 \AA / \mathrm{ps}, 120 \AA / \mathrm{ps}\right)$ are also given. It is found that the increase in Re is dramatic as the piston velocity increases from $10 \AA /$ ps to $40 \AA /$ ps (cases $1-3$ ), then slows down gradually when the piston velocity is higher than $40 \AA /$ ps. Thus, the instability growth in Fig. 3 can be concluded that 
TABLE II. Reynolds numbers for the present cases and previous experiments. $v_{0}$ is the growth rate at the linear stage, and $\mu$ and $\rho$ are the dynamic viscosity and density for the shocked fluid, respectively. The unit of dynamic viscosity is $\mathrm{kg} /(\mathrm{m} \mathrm{s})$.

\begin{tabular}{lcccccccc}
\hline \hline Case & $v_{p}(\AA / \mathrm{ps})$ & $\lambda(\AA)$ & $v_{0}(\AA / \mathrm{ps})$ & $\mu_{1}\left(\times 10^{-5}\right)$ & $\mu_{2}\left(\times 10^{-5}\right)$ & $\rho_{1}\left(\mathrm{~g} / \mathrm{cm}^{3}\right)$ & $\rho_{2}\left(\mathrm{~g} / \mathrm{cm}^{3}\right)$ & $\mathrm{Re}$ \\
\hline 1 & 10 & 800 & 0.73 & 7.1 & 33.9 & 0.44 & 1.68 & 1291 \\
2 & 15 & 800 & 0.96 & 9.5 & 42.5 & 0.51 & 1.83 & 1353 \\
3 & 20 & 800 & 1.34 & 11.8 & 48.1 & 0.57 & 1.94 & 1624 \\
4 & 40 & 800 & 2.50 & 22.1 & 73.6 & 0.70 & 2.24 & 1909 \\
5 & 80 & 800 & 4.78 & 44.4 & 121.2 & 0.82 & 2.54 & 2139 \\
6 & 150 & 800 & 8.49 & 93.0 & 209.2 & 0.91 & 2.80 & 2106 \\
& & & & & & & \\
Liu (2018) & $2.0 \times 10^{8}$ & 0.11 & 2.09 & 2.43 & $2.04 \times 10^{-3}$ & $8.46 \times 10^{-3}$ & 1204052 \\
Mariani (2008) & $8.1 \times 10^{8}$ & 0.08 & & & & & 3657811 \\
Jacobs (2002) & $7.3 \times 10^{8}$ & 0.04 & & & & & 1570165 \\
\hline \hline
\end{tabular}

for cases 1-3 with different Re, their perturbation growths exhibit an evident discrepancy, while for cases 4-6 with the nearly same Re, their amplitude histories collapse quite well. This finding implies a correlation between the Re and the instability growth, which is in coincidence with our physical intuition that the great viscosity in the present microscale RMI provides a strong resistance on the shear deformation and further inhibits the instability growth.

Variation of the ratio between the internal energy $\left(E_{p}\right)$ and the kinetic energy $\left(E_{k}\right)$ of the shocked fluid vs the velocity of piston is shown in Fig. 4. The $E_{p}$ is estimated as $E_{p}=3 N k_{b} T / 2$, where $\mathrm{N}$ is the total number of atoms and $k_{b}$ is the Boltzmann constant. The $E_{k}$ is calculated by $E_{k}=\mathrm{N} m v^{2} / 2$, with $m$ being the atom mass and $v$ being the post-shock velocity. It is seen that the value of $E_{p} / E_{k}$ drops gradually as the piston velocity increases from 10 to $40 \AA$ /ps (cases 1-3) but remains nearly invariant for cases 4-6. This is highly similar to the variation tendency of Re. As we know, the internal energy represents the microscopic behaviors, e.g., the viscosity,

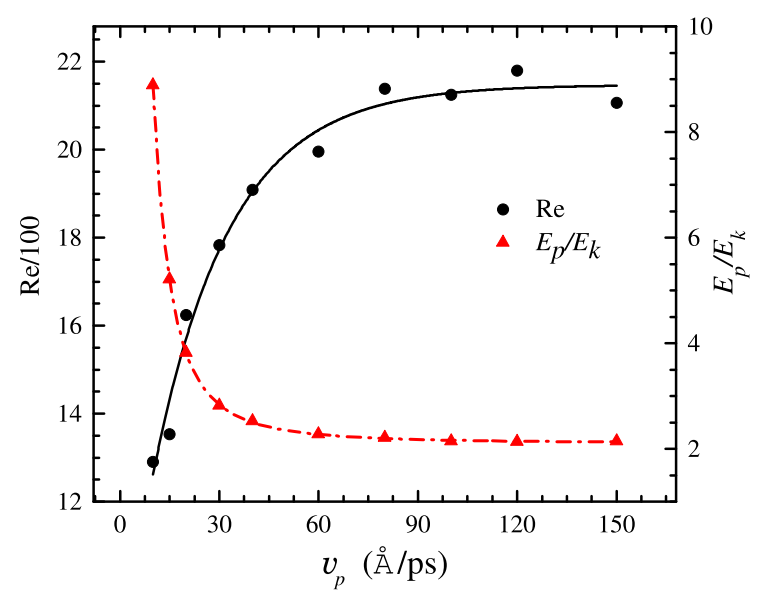

FIG. 4. Variations of the Reynolds number and the internal energy-to-kinetic energy ratio $\left(E_{p} / E_{k}\right)$ vs the piston velocity $\left(v_{p}\right)$. The $E_{p}$ is estimated as $E_{p}=3 N k_{b} T / 2$, where $\mathrm{N}$ is the total atom number and $k_{b}$ is the Boltzmann constant. The $E_{k}$ is calculated by $E_{k}=\mathrm{Nm} v^{2} / 2$, with $m$ being the atom mass and $v$ being the postshock velocity. and the kinetic energy indicates macroscopic dynamic motions such as the growth of perturbation amplitude. A much higher $E_{p} / E_{k}$ in cases $1-3\left(v_{p} \leq 20 \AA / \mathrm{ps}\right)$ means a stronger influence of molecular thermal motion on the macroscopic phenomena, which is in accordance with the greater viscosity effect observed in these cases. The present results illustrate the viscosity effect from molecular dynamic perspective.

\section{Theoretical considerations on growth rate in microscale RMI}

We first examine the linear growth of the present compressible viscous RMI. The linear growth rates for all cases, obtained by a linear fitting of the MD results, are listed in Table III, where the prediction of impulsive theory ${ }^{1}$ and the asymptotic growth rate of compressible linear theory ${ }^{41}$ are also given for comparison. The compressible linear theory, which is expected to give a good prediction, apparently overestimates the perturbation growth at a linear stage (especially for cases with stronger shocks) for the ignorance of the viscosity effect.

To characterize the RMI growth under the viscosity effect, two linear models considering the viscosity effect have been proposed by Mikaelian ${ }^{47}$ and Carlès and Popinet (CP), ${ }^{42}$ respectively. According to the comments of Mikaelian, ${ }^{43}$ the viscous model of Carlès and Popinet ${ }^{42}$ (CP) is merely a small-time expansion of the model of Mikaelian $^{47}$ (Mik) and thus performs slightly better in the linear stage even though it has a narrower validity range. Based on the $\mathrm{CP}$ model, here, a compressible linear model accounting for the viscous

TABLE III. The linear growth rates $(\AA / \mathrm{ps})$ from the MD results $\left(v_{M D}\right)$, the impulsive theory $\left(v_{\text {Rich }}\right),{ }^{1}$ the compressible linear model $\left(v_{\infty}^{c}\right),{ }^{41}$ and the compressible linear model considering the viscous damping $\left[\bar{v}_{\text {vis }}^{c}\right.$, see Eq. (8)].

\begin{tabular}{lcccccr}
\hline \hline Case & 1 & 2 & 3 & 4 & 5 & \multicolumn{1}{c}{6} \\
\hline$v_{M D}$ & 0.73 & 0.96 & 1.34 & 2.50 & 4.78 & 8.49 \\
$v_{\infty}^{c}$ & 0.93 & 1.35 & 1.79 & 3.50 & 6.78 & 12.42 \\
$\bar{v}_{\text {vis }}^{c}$ & 0.72 & 1.05 & 1.41 & 2.72 & 5.26 & 9.49 \\
$v_{\text {Rich }}$ & 0.76 & 1.06 & 1.36 & 2.51 & 4.78 & 8.71 \\
\hline \hline
\end{tabular}


damping is proposed, which is expressed as

$$
v_{v i s}^{c}=v_{\text {lin }}^{c}\left(1-\sqrt{\frac{128}{\operatorname{Re}}} \sqrt{\tau}\right) \text {. }
$$

Here, superscript " $c$ " denotes the compressible flow and subscripts "vis" and "lin" denote viscous and linear regimes, respectively.

Integrating Eq. (7) from 0 to $\tau_{1}$ and then dividing the time interval $\tau_{1}$, an averaged linear growth rate $\left(\bar{v}_{v i s}^{c}\right)$ for the compressible viscous RMI is available,

$$
\bar{v}_{v i s}^{c}=\left(\int_{0}^{\tau_{1}} v_{v i s}^{c} d \tau\right) / \tau_{1} .
$$

Here, $\tau_{1}=0.7$ is adopted for the limited range of linear regimes, as suggested in previous work. ${ }^{11}$ As shown in Table III, Eq. (8) provides a much better prediction of the MD results for the early-stage perturbation growth. It is surprising that the impulsive model, which ignores both the compressibility and viscosity effects, provides a quite good prediction of the linear growth. To illustrate the reason, time-varying growth rates predicted by the impulsive model, compressible linear model, and modified compressible linear model are shown in Fig. 5 (here only cases 2, 4, and 6 are given for a clear presentation). It is seen that for the present cases, the compressible linear theory predicts an asymptotic growth rate much higher than that of the impulsive model, and the modified compressible model prediction [Eq. (7)] considering the viscous damping is in fairly agreement with the impulsive theory. It illustrates that the compressibility effect, which promotes the linear growth for the present cases, can just compensate the viscosity effect, which inhibits the instability growth. This is a main reason for the reasonable prediction of the MD growth at the linear stage by the impulsive model ignoring both effects. It should be stressed that this agreement is just coincidental and the impulsive model may fail for other situations (e.g., in an air/ $\mathrm{SF}_{6}$ case where the compressibility causes an

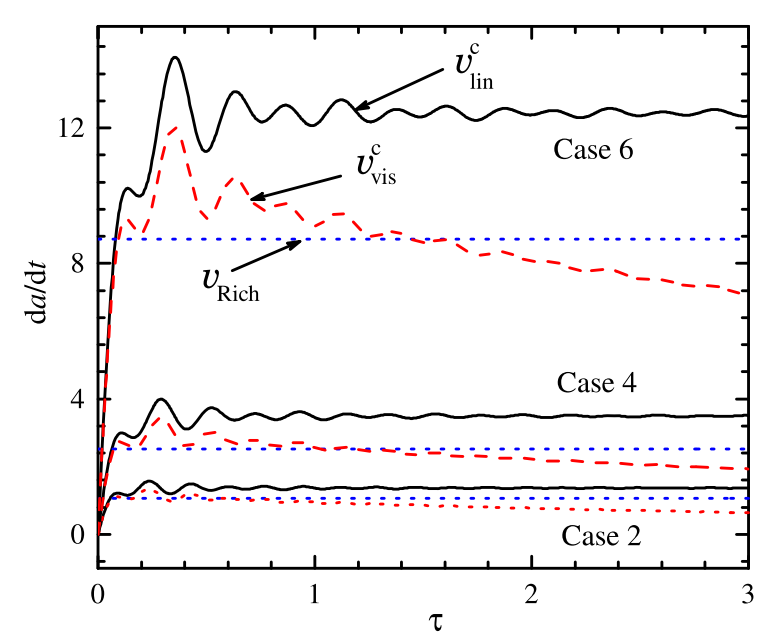

FIG. 5. The predicted growth rates for the impulsive model ( $v_{\text {Rich }}$, dotted line), ${ }^{1}$ the compressible linear model ( $v_{\text {lin }}^{c}$, solid line) ${ }^{41}$ and the compressible viscous model $\left[v_{\text {vis }}^{c}\right.$, dashed line, Eq. (7)]. asymptotic growth rate lower than the impulsive model prediction ${ }^{7}$ ). The present analysis indicates that for the microscopic RMI, three main flow regimes including baroclinic vorticity, viscosity, and compressibility dominate its linear growth, which is different from the macroscopic counterpart.

The CP and Mik models only considered the viscosity influence on the instability growth at the linear stage. In the nonlinear stage, higher harmonics emerge and their growths would also be damped by the viscosity. The evident viscosity effect on the nonlinear growth is revealed by the visible deviation of the present $\mathrm{MD}$ results from the prediction of Zhang et al. ${ }^{10}$ (see Fig. 3). Here, to quantify the viscosity effect on the whole-stage instability growth, the compressible nonlinear ZDG model [Eq. (5)] is combined with the theory of Mikaelian $^{47}$ (denoted as the ZDG-Mik model) and written as

$$
v_{n l}^{M i k}\left(t, A, \bar{v}_{v i s}^{c}\right)=v_{l i n}^{c}(t, A) P_{3}^{2}\left(t, A, \bar{v}_{v i s}^{c}\right) e^{-2 v k^{2} t} .
$$

As an alternative, the ZDG model can also be combined with the theory of Carlès and Popinet ${ }^{42}$ (called the ZDG-CP model),

$$
v_{n l}^{C P}\left(t, A, \bar{v}_{v i s}^{c}\right)=v_{\text {lin }}^{c}(t, A) P_{3}^{2}\left(t, A, \bar{v}_{v i s}^{c}\right)\left(1-b \sqrt{\frac{128}{\operatorname{Re}}} \sqrt{k \bar{v}_{v i s}^{c} t}\right) .
$$

Here, $b$ in Eq. (10) is a prefactor and equals 1.0 for the original $\mathrm{CP}$ model. Note that in both equations, the averaged linear growth rate of the compressible viscous model $\left(\bar{v}_{v i s}^{c}\right)$, instead of the original asymptotic growth rate of compressible theory $\left(v_{\infty}^{c}\right)$, is adopted as suggested by the above analysis on the instability growth at the linear stage.

Figure 6 compares the MD results with the predictions of the ZDG-Mik and ZDG-CP models. It is found that the ZDG-CP model gives a relatively more accurate prediction than the ZDG-Mik model even though both of them evidently underestimate the viscosity effect. It should be mentioned that the present ZDG-CP model has two inherent shortcomings, as pointed out by Mikaelian. ${ }^{43}$ First, it is applicable only at short times $t<t^{c p}$. Second, it cannot be used for systems where the viscosity coefficient of fluid at one side of the interface is zero or much smaller than that on the other side. The dimensionless critical times for cases $1-6$ are $\tau^{c p}=10.5,11.7$, $12.9,15.0,16.7$, and 16.9 , respectively, and the time range considered in this work $(\tau<3.0)$ is much shorter than the critical time. In addition, moderate density and viscosity ratios are employed for the present cases, which satisfies the application condition of the ZDG$\mathrm{CP}$ model. These reasonably explain the good performance of the ZDG-CP model here. For systems with very large density and viscosity ratios or instability growths suffering very long-term viscous dissipation, the ZDG-Mik model would be more appropriate.

As has been stated, the ZDG-CP model underestimates the viscous effect and, thus, one can improve the model by increasing the prefactor $b$ in Eq. (10). We find that a modified ZDG-CP model employing $b=1.25$ (called the m-ZDG-CP model) gives a fairly good prediction of the MD results from early to late nonlinear stages for all cases considered in this work, as shown in Fig. 7(a). In addition, the growths of the individual amplitudes of the bubble and spike are well predicted, as shown in Fig. 7(b). Furthermore, as shown in Fig. 8(a), the perturbation growth rates of the MD results are well reproduced by this improved model, which provides 
(a)

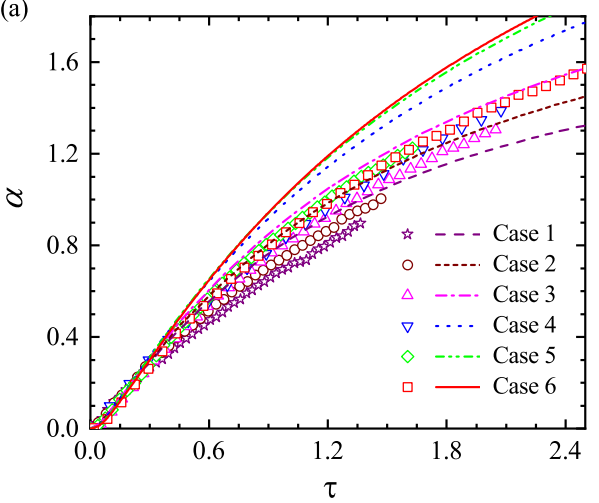

(a)

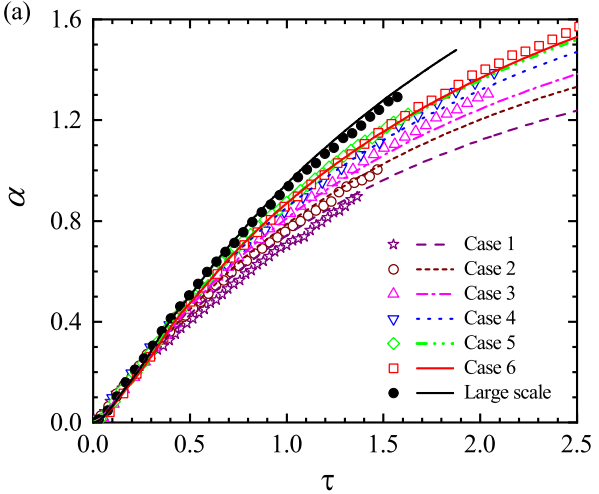

(b)

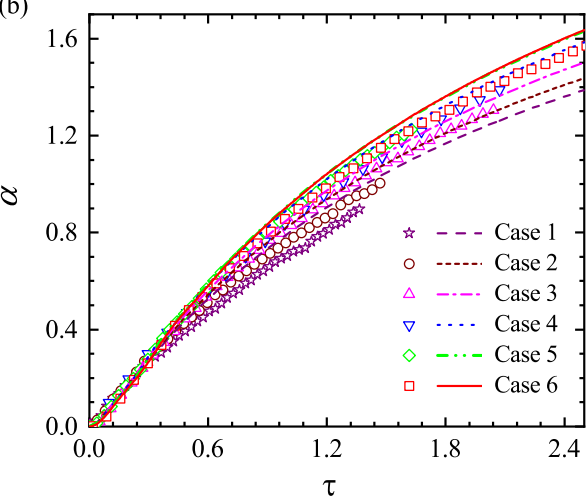

(b)

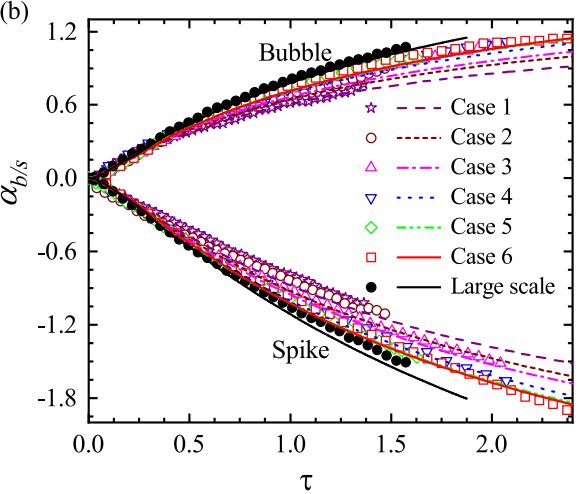

FIG. 6. Comparisons of the MD results with the predictions of (a) the ZDG-Mik model and (b) the ZDG-CP model for the overall perturbation amplitude growth. Symbols stand for the MD results and lines for the model predictions. a convincing validation of the model accuracy. Note that although the present $\mathrm{m}$-ZDG-CP model exhibits a good capacity in forecasting the compressible nonlinear viscous RMI with a broad range of $\mathrm{Re}$ and $\mathrm{Ma}$, it has the same limitations as those of the original CP model. ${ }^{42}$

In addition, we increase the flow Re by enlarging the characteristic scale (i.e., perturbation wavelength) rather than by intensifying the shock strength. Here, we take case 5 as an example, namely, the initial wavelength of perturbation in case 5 is enlarged twice, corresponding to an increment of Re from 2139 to 4278. As shown in Fig. 7 , for the larger-scale case 5, the viscosity effect becomes much weaker, leading to a delay of the growth saturation. The MD results are also well forecasted by the modified model. The present study suggests that for RMI flow with Re lower than $10^{5}$ [calculated based on Eq. (6)], viscous dissipation produces an evident suppression on the instability growth, which should be taken into account. It should be stated that the Re in previous shock-tube experiments (Table II), calculated based on Eq. (6) utilizing the corresponding flow data, is much higher than the critical Re and, thus, the viscosity effect can be negligible. This reasonably explains the considerable accuracy of the original ZDG model in forecasting the macroscopic RMI.
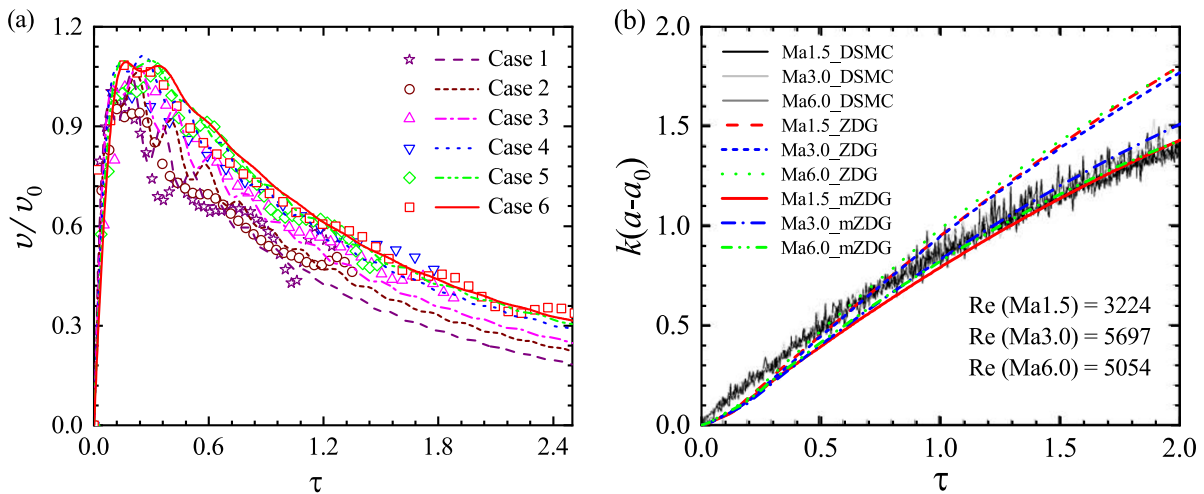

FIG. 8. (a) Comparisons between the MD simulation (symbols) and the m-ZDG$\mathrm{CP}$ model prediction (lines) for the perturbation growth rate. (b) Comparison of the DSMC simulation results by Gallis et $a .^{23}$ with the predictions of $Z D G$ and m-ZDG-CP models. 
To further demonstrate the model accuracy, the results of the DSMC simulation for different shock strengths from Gallis et al. ${ }^{23}$ are compared against the predictions of the new model, as shown in Fig. 8(b). As expected, the original ZDG model evidently overestimates the DSMC results, while the $\mathrm{m}-\mathrm{ZDG}-\mathrm{CP}$ model involving appropriate viscous dissipation gives a fairly accurate forecast of the instability growth, which makes the new model more convincing. Note that the DSMC simulation has a larger spatial scale than the present MD simulation and the Re increases slowly as the shock Mach number increases from 1.5 to 6.0. This indicates that the viscous damping effect changes slightly with intensifying the incident shock, which reasonably explains the collapse of the DSMC results for three cases. The m-ZDG-CP model also well reproduces such a collapse. The present finding illustrates the significant role of the viscosity effect in RMI flows at a small scale (low Re). We stress again that the viscosity effect is a primary reason for the different dependence of the perturbation growth on the shock intensity for the present MD results. This is different from the Mach number scaling laws for strong shocks reported by Zhang and $\mathrm{Graham}^{38}$ and Samtaney and Meiron. ${ }^{48}$ The m-ZDG-CP and ZDG-Mik models considering the compressibility, nonlinearity, and viscosity effects would be useful for the further research on RMI.

There are a few experimental works on microscopic or high Mach number RMI published before. ${ }^{12,13,49}$ It is found that there is a significant difference between the previous experiments and the present MD simulation for the initial settings. First, in those experiments, the initial shock wave is produced by the laser irradiation on the OMEGA or NOVA facility and, thus, the post-shock flow condition is different from the counterpart produced by a moving piston adopted in this work. Second, in experiments, the RMI is examined in a cylindrical geometry. As has been demonstrated recently, ${ }^{50}$ the flow regimes of cylindrical RMI are much more complicated than those of planar RMI due to the presence of geometric contraction and Rayleigh-Taylor effect. Therefore, it is difficult to give a quantitative comparison between the present MD results and those of experiments.

\section{CONCLUSIONS}

The microscopic-scale RMI of a single-mode light/heavy gaseous interface subjected to a planar shock is studied via the MD approach. Physically realistic evolution processes involving the nonequilibrium effects such as diffusion, dissipation, and thermal conduction are obtained for various shock strengths, which are crucial for ICF application but cannot be studied via the traditional hydrodynamic method. Different dependence of the perturbation growth on the shock intensity is observed. Specifically, amplitude growths for cases 1-3 with lower shock Mach numbers $(\mathrm{Ma}=1.9,2.4,2.9)$ exhibit an evident discrepancy from a very early stage, whereas for cases 4-6 with stronger shocks $(\mathrm{Ma}=4.9,9.0,16.0)$, their amplitude variations with time match quite well during the whole evolution time. Such different behaviors are ascribed to the viscosity effect that plays an essential role in the microscale RMI.

To characterize the viscosity effect, a new Reynolds number is defined based on the characteristic quantities of RMI flow. The compressible linear theory of Yang et al. ${ }^{41}$ accounting for the viscosity dissipation provides a reasonable prediction of the linear growth rate. It is found that the compressibility effect, which promotes the linear growth for the present cases, can just offset the viscosity effect, which inhibits the instability growth. As a result, the impulsive model of Richtmyer, ${ }^{1}$ which ignores both effects, can reasonably forecast the linear growth.

Furthermore, a modified model of Zhang et al. ${ }^{10}$ accounting for both the viscosity (Reynolds number) effect and the linear prediction correction is proposed, which gives an excellent forecast of the microscopic RMI from early to late nonlinear stages no matter if for the whole perturbation amplitude or for the growth rate. This modified model is further validated by the DSMC results of Gallis et al. ${ }^{23}$ Note that the model is applicable only to the instability growth at short times and to situations where the viscosity ratio is not very large. The present finding provides deep insights into the RMI at small scales and may be helpful for ICF capsule design.

\section{ACKNOWLEDGMENTS}

This work was supported by the National Natural Science Foundation of China (Grant Nos. 11802304, 11625211, and 11621202), the Science Challenging Project (No. TZ2016001), and the National Key R\&D Program of China (Grant No. 2016YFC0800100). The numerical calculations in this paper were made on the supercomputing system in the Supercomputing Center of University of Science and Technology of China.

\section{REFERENCES}

${ }^{1}$ R. D. Richtmyer, "Taylor instability in shock acceleration of compressible fluids," Commun. Pure Appl. Math. 13, 297-319 (1960).

${ }^{2}$ E. E. Meshkov, "Instability of the interface of two gases accelerated by a shock wave," Fluid Dyn. 4, 101-104 (1969).

${ }^{3}$ J. Lindl, O. Landen, J. Edwards, E. Moses, and N. Team, "Review of the national ignition campaign 2009-2012," Phys. Plasmas 21, 020501 (2014).

${ }^{4}$ C. C. Kuranz, H. S. Park, C. M. Huntington, A. R. Miles, B. A. Remington, T. Plewa, M. R. Trantham, H. F. Robey, D. Shvarts, A. Shimony et al., "How high energy fluxes may affect Rayleigh-Taylor instability growth in young supernova remnants," Nat. Commun. 9, 1564 (2018).

${ }^{\mathbf{5}}$ J. G. Wouchuk, "Growth rate of the linear Richtmyer-Meshkov instability when a shock is reflected," Phys. Rev. E 63, 056303 (2001).

${ }^{6} \mathrm{X}$. Luo, X. Wang, and T. Si, "The Richtmyer-Meshkov instability of a threedimensional air $/ \mathrm{SF}_{6}$ interface with a minimum-surface feature," J. Fluid Mech. 722, R2 (2013).

${ }^{7}$ Q. Zhang and S. I. Sohn, "Nonlinear theory of unstable fluid mixing driven by shock wave," Phys. Fluids 9, 1106-1124 (1997).

${ }^{8}$ O. Sadot, L. Erez, U. Alon, D. Oron, L. A. Levin, G. Erez, G. Ben-Dor, and D. Shvarts, "Study of nonlinear evolution of single-mode and two-bubble interaction under Richtmyer-Meshkov instability," Phys. Rev. Lett. 80, 1654-1657 (1998).

${ }^{9}$ G. Dimonte and P. Ramaprabhu, "Simulations and model of the nonlinear Richtmyer-Meshkov instability,” Phys. Fluids 22, 014104 (2010).

${ }^{10} \mathrm{Q}$. Zhang, S. Deng, and W. Guo, "Quantitative theory for the growth rate and amplitude of the compressible Richtmyer-Meshkov instability at all density ratios," Phys. Rev. Lett. 121, 174502 (2018).

${ }^{11}$ L. Liu, Y. Liang, J. Ding, N. Liu, and X. Luo, "An elaborate experiment on the single-mode Richtmyer-Meshkov instability,” J. Fluid Mech. 853, R2 (2018).

${ }^{12}$ J. R. Fincke, N. E. Lanier, S. H. Batha, R. M. Hueckstaedt, G. R. Magelssen, S. D. Rothman, K. W. Parker, and C. J. Horsfield, "Postponement of saturation of the Richtmyer-Meshkov instability in a convergent geometry," Phys. Rev. Lett. 93, 115003 (2004).

${ }^{13}$ J. R. Fincke, N. E. Lanier, S. H. Batha, R. M. Hueckstaedt, G. R. Magelssen, S. D. Rothman, K. W. Parker, and C. J. Horsfield, "Effect of convergence on growth of the Richtmyer-Meshkov instability," Laser Part. Beams 23(01), 21-25 (2005). 
${ }^{14}$ H. Lai, A. Xu, G. Zhang, Y. Gan, Y. Ying, and S. Succi, "Nonequilibrium thermohydrodynamic effects on the Rayleigh-Taylor instability in compressible flows," Phys. Rev. E 94, 023106 (2016)

${ }^{15}$ V. V. Zhakhovskii, S. V. Zybin, S. I. Abarzhi, and K. Nishihara, "Atomistic dynamics of Richtmyer-Meshkov instability in cylindrical and planar geometries," AIP Conf. Proc. 845, 433-436 (2006).

${ }^{16}$ S. V. Zybin, V. V. Zhakhovskii, E. M. Bringa, S. I. Abarzhi, and B. Remington, "Molecular dynamics simulations of the Richtmyer-Meshkov instability in shock loaded solids," AIP Conf. Proc. 845, 437-441 (2006).

${ }^{17}$ T. C. Germann, G. Dimonte, J. E. Hammerberg, K. Kadau, J. Quenneville, and M. B. Zellner, "Large-scale molecular dynamics simulations of particulate ejection and Richtmyer-Meshkov instability development in shocked copper," EDP Sci. 2, 1499-1505 (2009).

${ }^{18}$ H. Liu, W. Kang, Q. Zhang, Y. Zhang, H. Duan, and X. T. He, "Molecular dynamics simulations of microscopic structure of ultra strong shock waves in dense helium," Front. Phys. 11, 115206 (2016).

${ }^{19} \mathrm{~S}$. Huang, W. Wang, and X. Luo, "Molecular-dynamics simulation of Richtmyer-Meshkov instability on a $\mathrm{Li}-\mathrm{H}_{2}$ interface at extreme compressing conditions," Phys. Plasmas 25, 062705 (2018).

${ }^{20}$ F. J. Cherne, J. E. Hammerberg, M. J. Andrews, V. Karkhanis, and P. Ramaprabhu, "On shock driven jetting of liquid from non-sinusoidal surfaces into a vacuum," J. Appl. Phys. 118, 185901 (2015).

${ }^{21}$ G. Dimonte, G. Terrones, F. J. Cherne, and P. Ramaprabhu, "Ejecta source model based on the nonlinear Richtmyer-Meshkov instability," J. Appl. Phys. 113, 024905 (2013)

${ }^{22}$ J. L. Barber, K. Kadau, T. C. Germann, and B. J. Alder, "Simulation of fluid instabilities using atomistic methods," AIP Conf. Proc. 955, 301-304 (2007).

${ }^{23}$ M. A. Gallis, T. P. Koehler, J. R. Torczynski, and S. J. Plimpton, "Direct simulation Monte Carlo investigation of the Richtmyer-Meshkov instability," Phys. Fluids 27, 084105 (2015).

${ }^{24}$ M. Brouillette, "The Richtmyer-Meshkov instability," Annu. Rev. Fluid Mech. 34, 445-468 (2002).

${ }^{25}$ D. Ranjan, J. Oakley, and R. Bonazza, "Shock-bubble interactions," Annu. Rev. Fluid Mech. 43, 117-140 (2011).

${ }^{26} \mathrm{Y}$. Zhou, "Rayleigh-Taylor and Richtmyer-Meshkov instability induced flow, turbulence, and mixing. I," Phys. Rep. 720-722, 1-136 (2017).

${ }^{27}$ S. Plimpton, "Fast parallel algorithms for short-range molecular dynamics," J. Comput. Phys. 117, 1 (1995).

${ }^{28}$ B. Li, L. Wang, J. C. E, H. H. Ma, and S. N. Luo, "Shock response of He bubbles in single crystal Cu," J. Appl. Phys. 116, 213506 (2014).

${ }^{29} \mathrm{Z}$. Wu, S. Huang, J. Ding, W. Wang, and X. Luo, "Molecular dynamics simulation of cylindrical Richtmyer-Meshkov instability," Sci. China Phys., Mech. Astron. 61, 114712 (2018).

${ }^{30} \mathrm{O}$. Talu and A. L. Myers, "Reference potentials for adsorption of helium, argon, methane, and krypton in high-silica zeolites," Colloids Surf., A 187, 83 (2001).

${ }^{31} \mathrm{M}$. Schoen, R. Vogelsang, and C. Hoheise, "Computation and analysis of the dynamic structure factor S $(k, \omega)$ for small wave vectors," Mol. Phys. 57, 445-471 (1986).
${ }^{32} \mathrm{~J}$. W. Jacobs and V. V. Krivets, "Experiments on the late-time development of single-mode Richtmyer-Meshkov instability," Phys. Fluids 17, 034105 (2005).

${ }^{33}$ C. Mariani, M. Vandenboomgaerde, G. Jourdan, D. Souffland, and L. Houas, "Investigation of the Richtmyer-Meshkov instability with stereolithographed interfaces," Phys. Rev. Lett. 100, 254503 (2008).

${ }^{34}$ O. Schilling, M. Latini, and W. S. Don, "Physics of reshock and mixing in singlemode Richtmyer-Meshkov instability," Phys. Rev. E 76, 026319 (2007).

${ }^{35} \mathrm{P}$. Movahed and E. Johnsen, "A solution-adaptive method for efficient compressible multifluid simulations, with application to the Richtmyer-Meshkov instability," J. Comput. Phys. 239, 166-186 (2013).

${ }^{36}$ O. Sadot, A. Rikanati, D. Oron, G. Ben-Dor, and D. Shvarts, “An experimental study of the high Mach number and high initial-amplitude effects on the evolution of the single-mode Richtmyer-Meshkov instability," Laser Part. Beams 21, 341-346 (2003).

${ }^{37}$ J. A. Aguilera and C. Aragón, "A comparison of the temperatures and electron densities of laser-produced plasmas obtained in air, argon, and helium at atmospheric pressure,” Appl. Phys. A 69, S475-S478 (1999).

${ }^{38} \mathrm{Q}$. Zhang and M. J. Graham, "Scaling laws for unstable interfaces driven by strong shocks in cylindrical geometry," Phys. Rev. Lett. 79, 2674-2677 (1997).

${ }^{39}$ D. Ranjan, J. Niederhaus, B. Motl, M. Anderson, J. Oakley, and R. Bonazza, "Experimental investigation of primary and secondary features in high-Machnumber shock-bubble interaction," Phys. Rev. Lett. 98, 024502 (2007).

${ }^{40}$ G. C. Orlicz, S. Balasubramanian, and K. P. Prestridge, "Incident shock Mach number effects on Richtmyer-Meshkov mixing in a heavy gas layer," Phys. Fluids 25, 114101 (2013).

${ }^{41}$ Y. Yang, Q. Zhang, and D. H. Sharp, "Small amplitude theory of RichtmyerMeshkov instability,” Phys. Fluids 6(5), 1856-1873 (1994).

${ }^{42} \mathrm{P}$. Carlès and S. Popinet, "Viscous nonlinear theory of Richtmyer-Meshkov instability," Phys. Fluids 13, 1833 (2001).

${ }^{43} \mathrm{~K}$. O. Mikaelian, "Comment on "The effect of viscosity, surface tension and nonlinearity on Richtmyer-Meshkov instability" [Eur. J. Mech. B Fluids 21 (2002) 511-526]," Eur. J. Mech. B: Fluids 43, 183-184 (2014).

${ }^{44}$ R. Samtaney and N. J. Zabusky, "Circulation deposition on shock-accelerated planar and curved density-stratified interfaces: Models and scaling laws," J. Fluid Mech. 269, 45-78 (1994).

${ }^{45}$ R. Samtaney, J. Ray, and N. J. Zabusky, "Baroclinic circulation generation on shock accelerated slow/fast gas interfaces," Phys. Fluids 10, 1217-1230 (1998).

${ }^{46} \mathrm{~B}$. Walchli and B. Thornber, "Reynolds number effects on the single-mode Richtmyer-Meshkov instability," Phys. Rev. E 95, 013104 (2017).

${ }^{47}$ K. O. Mikaelian, "Effect of viscosity on Rayleigh-Taylor and RichtmyerMeshkov instabilities," Phys. Rev. E 47, 375-383 (1993).

${ }^{48}$ R. Samtaney and D. I. Meiron, "Hypervelocity Richtmyer-Meshkov instability," Phys. Fluids 9, 1783 (1997).

${ }^{49}$ J. F. Hansen, H. F. Robey, R. I. Klein, and A. R. Miles, "Experiment on the massstripping of an interstellar cloud in a high Mach number post-shock flow," Phys. Plasmas 14(5), 056505 (2007).

${ }^{50}$ J. Ding, T. Si, J. Yang, X. Lu, Z. Zhai, and X. Luo, "Measurement of a RichtmyerMeshkov instability at an air-SF $\mathrm{SF}_{6}$ interface in a semiannular shock tube," Phys. Rev. Lett. 119, 014501 (2017). 